\title{
Development of a New Recurrence-Free Survival Prediction Nomogram for Patients with Primary Non-Muscle-Invasive Bladder Cancer Based on Preoperative Controlling Nutritional Status Score
}

\author{
Liwei Zhao ${ }^{1,2, *}$ \\ Ji Sun ${ }^{2, *}$ \\ Kai Wang ${ }^{3}$ \\ Shengcheng $\mathrm{Tai}^{2}$ \\ Runmiao Hua ${ }^{2}$ \\ Yufu Yu ${ }^{2}$ \\ Yi Fan ${ }^{1,2}$ \\ Jiaguo Huang ${ }^{2}$ \\ 'Department of Urology, School of \\ Medicine, Hangzhou Normal University, \\ Hangzhou, 3III2I, Zhejiang Province, \\ People's Republic of China; ${ }^{2}$ Department \\ of Urology, Affiliated Xiaoshan Hospital, \\ Hangzhou Normal University, Hangzhou, \\ 3I I 202, Zhejiang Province, People's \\ Republic of China; ${ }^{3}$ Department of \\ Urology, Affiliated Hangzhou First \\ People's Hospital, Zhejiang University \\ School of Medicine, Hangzhou, 310006 \\ Zhejiang Province, People's Republic of \\ China
}

*These authors contributed equally to this work
Correspondence: Yi Fan; Jiaguo Huang Department of Urology, Affiliated Xiaoshan Hospital, Hangzhou Normal University, No. 728 North Yucai Road, Xiaoshan District, Hangzhou, 3 I I202, Zhejiang, People's Republic of China Tel +86-57l-83865858

Email fanyi316@sina.com;

13735526508@163.com
Background: Bladder cancer is the second most prevalent neoplasm in the urogenital system in terms of morbidity and mortality, and there is an urgent need for a more accurate assessment of individual prognosis in patients with primary non-muscle-invasive bladder cancer (NMIBC). The Controlling Nutritional Status (CONUT) score is an emerging biomarker score which has been confirmed to have prognostic value in various malignant tumors. The study attempted to systematically identify the prognostic role of preoperative CONUT score on posttreatment recurrence-free survival (RFS) in patients with NMIBC, and determine the predictive value and feasibility of the new prognostic prediction model.

Methods: A total of 94 patients with NMIBC were analyzed retrospectively between January 2011 and December 2015. Statistical analysis was conducted using the nonparametric method. The Kaplan--Meier method was used to assess recurrence-free survival (RFS), and Log rank tests was used to analyze the equivalences of survival curves. We used univariate and multivariate Cox proportional hazards regression model to identify important predictors of RFS. Discrimination of nomogram was measured by the concordance index. Predictive accuracy of the model was evaluated using the internal validation.

Results: In univariate analysis, age, history of smoking, pathological $\mathrm{T}$ stage, tumor grade, tumor size, and CONUT score were significantly correlated with RFS. Multivariate analysis indicated that CONUT score (HR $=3.855,95 \%$ CI 1.242-11.970, $\mathrm{p}=0.020$ ) was an independent predictor of RFS in patients with NMIBC. Based on significant parameters in multivariate analysis and reliable recurrence predictors determined in predictive models and relevant guidelines, a new age-, history of smoking-, pathologic factors- and the CONUT score-based scoring model was developed to predict recurrence of NMBIC. In addition, we internally validated the nomogram using the consistency index and calibration plots, which demonstrated that the model has high prediction accuracy (c-index=0.851).

Conclusion: The development of a new nomogram based on CONUT score could increase the accuracy of recurrence prediction and improve individualized treatment plans for patients with NMIBC.

Keywords: bladder cancer, CONUT score, nomogram, the EORTC scoring system, recurrence-free survival 


\section{Introduction}

Bladder cancer ranks 11th among the most prevailing cancers in the world. The global age-standardized incidence per 100,000 person-years is 9.0 for men and 2.2 for women, ${ }^{1}$ and it is the sixth most common cancer in males and the seventeenth in females, respectively. ${ }^{2}$ It is also the most common malignancy of the urinary tract. ${ }^{3}$ More than $90 \%$ of bladder cancer cases are urothelial carcinomas, and approximately $75-85 \%$ of patients present as NMIBC, which is defined as confined to the mucosa (carcinoma in situ and stage Ta) or submucosa (stage T1) ${ }^{1,4}$ Although transurethral resection of the bladder tumor (TURBT) or partial cystectomy can completely resect $\mathrm{Ta}$ and $\mathrm{T} 1$ tumors, it is a heterogeneous disease with recurrence rates following treatment, and even advances to the muscle as an invasive and metastatic tumor. It is reported that the 5-year recurrence rate of NMIBC is 50 $70 \%$, and the 5 -year progression rate is $10-30 \%{ }^{5}$

It is important to control the progression of NMIBC due to a poor prognosis in patients with metastatic bladder cancer. Although some studies have shown that recurrence is related to factors including surgical precision levels, drug infusion, lifestyle and chemical contact, there is still no effective avenue to reduce the recurrence rate of bladder cancer. ${ }^{6,7}$ The treatment of recurrent bladder cancer focuses on early detection and treatment. Therefore, prognostic and risk assessments are essential for treatment decision-making and patient consultation.

Nomogram is a widely used statistical instrument for predicting the prognosis of individuals by calculating the scores of numerous variables, and it was first used to predict of recurrence in patients with NMIBC by Hong et al. ${ }^{8}$ The two scoring models of The European Organization for Research and Treatment of Cancer (EORTC) and The Spanish Urology Association for Oncological Treatment (CUETO) are the most commonly used individual prognostic models of bladder cancer, which have certain clinical value, though also have a set of shortcomings, ${ }^{9}$ such as lacking in the degree of hematological indexes. Consequently, we need to develop a more precise predictive model for patients with NMIBC by using the nomogram.

Recently, a number of studies have reported that nutritional status and systemic inflammatory response have an impact on the prognosis of cancer patients. Malnutrition usually manifested as hypoalbuminemia, which is associated with poor prognosis of various malignant tumors, and the presence of persistent inflammation is considered as one of the hallmarks of cancer. ${ }^{11}$ As a novel nutritional screening tool, the Controlling Nutritional Status (CONUT) score is a cumulative score calculated from serum albumin level, total cholesterol level, and total peripheral blood lymphocyte count measurement, reflecting both the nutritional and immune status of the subjects. The scoring system is readily available and significantly correlates with both Subjective Global Assessment (SGA) and Full Nutritional Assessment (FNA). ${ }^{12}$ In addition, it has been reported to be associated with the postoperative complications and the prognosis of various malignant tumors. $^{13-17}$

Presently, the predictive value of CONUT for RFS in patients with NMIBC has not been explored. Therefore, we would like to clarify the prognostic value of the preoperative CONUT score in patients who have undergone TURBT or partial cystectomy following initial diagnosis of NMIBC, and to develop a novel model to predict the postoperative RFS for individual patients based on the CONUT score.

\section{Patients and Methods}

\section{Patient Selection}

We retrospectively reviewed the clinic-pathological and follow-up data of 94 newly diagnosed NMIBC patients who underwent initial TURBT or partial cystectomy in our hospital from January 2011 to December 2015. Patients enrolled in the study met the following criteria: (1) complete data of serum albumin concentration, total cholesterol concentration and total peripheral lymphocyte count prior to surgery were available; (2) the primary diagnosis was NMIBC, and the pathological staging was Ta or T1 tumor without carcinoma in situ (CIS); (3) there were no other systemic autoimmune diseases or cancers, and no previously received neoadjuvant chemotherapy or radiotherapy; (4) preoperative exclusion of distant metastasis from the tumor, and (5) complete follow-up data were available. In accordance with the guidelines of the European Association of Urology (EAU), the treatment protocol was discussed for each patient. The study was conducted according to the Declaration of Helsinki (2013 revision), written informed consent was obtained from all participating patients in this study and stored in our hospital database. 
The research was approved by the Institutional Ethics Committee of our hospital.

\section{Data Collection and Patient Follow-Up Data Collection}

All patients received routine hematological examination, computed tomography, transabdominal ultrasound, urine cytology or tissue biopsy, cystoscopy prior to surgery in order to diagnose NMIBC, and finally confirmed by postoperative pathology. Pre-operative baseline clinical pathology and laboratory data such as age, gender, body mass index (BMI), smoking history, tumor size, pathological $\mathrm{T}$ stage, and grade were obtained through electronic medical records in the hospital and reviewed. All histopathology reports were based on the eighth edition of Tumor-NodeMetastasis staging system, and the grade was assessed based on the 2004 WHO grading system. ${ }^{18,19}$ Tumor size was the sum of the longest diameters of all postoperative pathological specimens. According to the blood test results (within one month prior to surgery), the preoperative serum albumin concentration, total cholesterol concentration and total peripheral lymphocyte count of all individuals were used to calculate the CONUT score. Based on the previous study, ${ }^{12}$ the scoring criteria of CONUT are illustrated in Table 1.

\section{Follow-Up}

The deadline for follow-up was December 2015. Due to the low mortality of NMIBC, we were unable to use overall survival as the study end point, so we selected RFS as an indicator for endpoint evaluation in the study, which is defined as the time between the first TURBT or partial cystectomy and histopathological confirmation of recurrence. The follow-up period for each patient began following the radical surgery and ended with histologically confirmed tumor recurrence or deadline. All patients were followed up regularly according to the standard. During the follow-up period, routine urine test, routine blood test, biochemical test and cystoscopy were performed once every three months for the first two years, followed by 6-month intervals for the next three years and annual examination ( $>5$ years). An excretion urography or computed tomography scan was performed annually for five years after surgery.

\section{The EORTC-GUCG Risk Scoring System}

Currently, the most commonly used model for predicting outcomes in individual patients with bladder cancer is the EORTC-GUCG risk scoring system. The scoring system was based on $2596 \mathrm{TaT} 1$ tumor patients and evaluated from 6 clinical pathological factors: tumor size, number of tumors, previous recurrence rate, $\mathrm{T}$ category, tumor grade and CIS. According to the disease recurrence and progression risk scoring system, risk is divided into the following three layers: (1) Low-risk: primary, single, < $3 \mathrm{~cm}$, Ta stage, low grade or G1 grade, and no CIS; (2) Intermediate-risk: undetermined tumors in the two adjacent categories (between low-risk and high-risk categories); (3) High-risk: T1 stage tumors, high grade or G3 grade tumors, presence of CIS, and large $(>3 \mathrm{~cm})$, multiple, relapsed, Ta G1or G2 grade tumors (All conditions must be met simultaneously at this time) ${ }^{20}$

\section{Statistical Analyses}

In this study, the mean and standard deviation (SD) were used to describe continuous variables. Categorical variables were compared employing Pearson's chi-square test or Fisher's exact test. Possible cut-off values for the CONUT score were determined by applying receiver operating curve (ROC) analysis, as defined by the most significant point on the ROC curve (when Youden index is at its maximum) and calculating the area under the ROC (AUROC) curve. Further analysis was performed by using the optimal cut-off value. Concerning RFS analysis, evaluating the survival rate in different groups was used by Kaplan-Meier method, and the Log rank test was used to analyze the equivalences of the survival curves. Univariate and multivariate analyses were performed using Cox

Table I The Scoring System for the Controlling Nutritional Status (CONUT) Scale

\begin{tabular}{|l|l|l|l|l|}
\hline Undernutrition Degree & None & Light & Moderate & Severe \\
\hline Serum albumin $(\mathrm{g} / \mathrm{dL})$ & $\geq 3.5$ & $3.0-3.49$ & $2.5-2.99$ & $<2.5$ \\
Score & 0 & 2 & 4 & 6 \\
Total lymphocyte count $\left(/ \mathrm{mm}^{3}\right)$ & $\geq 1600$ & $\mid 200-1599$ & $800-1199$ & $<800$ \\
Score & 0 & 1 & 2 & 3 \\
Total cholesterol $(\mathrm{mg} / \mathrm{dL})$ & $\geq 180$ & $140-179$ & $100-139$ & 3 \\
Score & 0 & 1 & 2 & \\
\hline
\end{tabular}


proportional hazards regression model, and a nomogram predicting RFS across 1-, 3- and 5-year were constructed, based on the multivariable model. Several risk factors for recurrent NMBIC, identified in the guidelines and previous models, were added to the nomogram.

The nomogram provided an association between the multivariate prognostic factors of patients and the probability of RFS in patients with NMIBC by graphical representation. The discrimination was measured using the consistency index (c-index). A score of 1 implies perfect predictions, and a score of 0.5 indicates that the model has no discriminative ability. The model calibration is evaluated visually with the calibration plots. When the predictive values of the model perfectly match the actual risks of the patient, a $45^{\circ}$ line represents perfect calibration. Above or below the $45^{\circ}$ line indicates a deviation from that prediction. Our analysis used two-sided p-values, with a two-sided p-value $<0.05$ was considered to be statistically significant. All statistical analyses were performed using the MedCalc ${ }^{\circledR}$ [version 19.5.1; Ostend, Belgium], the Statistical Product and Service Solutions ${ }^{\circledR}$ [SPSS, version 26.0; IBM Corporation ${ }^{\mathrm{TM}}$, Chicago, IL, USA] software and $\mathrm{R}^{\circledR}$ [version 4.0.3; R Foundation, Vienna, Austria].

\section{Results}

\section{Basic Clinical Data of Patients}

From January 2011 to December 2015, among 116 patients with NMIBC who underwent TURBT or partial cystectomy for the first time in our hospital, 22 of them were excluded as they did not meet the inclusion criteria. The mean follow-up time was 43.61 (21.93) months. The clinical data of 94 patients are illustrated in Table 2. There were 75 males $(79.79 \%)$ and 19 females $(21.93 \%)$, with an average age (SD) of 63.22 (13.28) years old. Among these patients, 52 (55.32\%) had a history of smoking and the number of patients with $\mathrm{BMI} \geq 24$ and $<24$ was 37 (39.36\%) and 57 $(60.64 \%)$, respectively. Tumor grade was low grade in $58(61.70 \%)$ and high grade in $36(38.30 \%)$ patients. Pathological T-category was Ta in 67 (71.28\%) and T1 in $27(28.72 \%)$ patients. The primary tumor size was lower than $3 \mathrm{~cm}$ in 57 patients $(60.64 \%)$ and equal and/ or greater than $3 \mathrm{~cm}$ in 37 patients (39.34\%). The CONUT score was 0 in 26 patients $(27.66 \%), 1$ in 23 patients $(24.47 \%), 2$ in 22 patients $(23.40 \%), 3$ in 12 patients $(12.77 \%), 4$ in 6 patients $(6.38 \%), 5$ in 2 patients $(2.13 \%)$, and 6 in 3 patients $(3.19 \%)$, respectively. The recurrence rate was 33/94 (35.11\%) after five years of follow-up.

The relationships between the EORTC-GUCG risk scoring system and clinicopathological parameters of patients were stratified according to the EORTCGUCG risk scoring system, whereby there were 41 cases with low recurrence risk, 18 cases with intermediate recurrence risk and 35 cases with high recurrence risk among the 94 patients. The relationships between the EORTC-GUCG risk scoring system and clinicopathological parameters of patients are presented in Table 3. There were significant differences in patients' age and pathological factors among the varying risk groups.

Table 2 Patient Characteristics

\begin{tabular}{|c|c|}
\hline Factors & Value or Number of Patients(n=94) \\
\hline \multicolumn{2}{|l|}{ Age(years) } \\
\hline Mean $\pm S D$ & $63.22 \pm 12.28$ \\
\hline Range & $25-84$ \\
\hline \multicolumn{2}{|l|}{ Gender, n(\%) } \\
\hline Male & 75(79.79) \\
\hline Female & $19(20.21)$ \\
\hline \multicolumn{2}{|l|}{ BMI, n(\%) } \\
\hline$<24$ & $57(60.64)$ \\
\hline$\geq 24$ & $37(39.36)$ \\
\hline History of smoking, n(\%) & $52(55.32)$ \\
\hline \multicolumn{2}{|l|}{ Pathological T stage, n(\%) } \\
\hline pTa & $67(71.28)$ \\
\hline $\mathrm{pTI}$ & $27(28.72)$ \\
\hline \multicolumn{2}{|l|}{ Tumor grade, n(\%) } \\
\hline LG & $58(61.70)$ \\
\hline HG & $36(38.30)$ \\
\hline \multicolumn{2}{|l|}{ Tumor size, $n(\%)$} \\
\hline$<3 \mathrm{~cm}$ & $57(60.64)$ \\
\hline$\geq 3 \mathrm{~cm}$ & $37(39.36)$ \\
\hline \multicolumn{2}{|l|}{ CONUT score, n(\%) } \\
\hline 0 & $26(27.66)$ \\
\hline 1 & $21(24.47)$ \\
\hline 2 & $20(23.40)$ \\
\hline 3 & $12(12.77)$ \\
\hline 4 & $5(6.38)$ \\
\hline 5 & $\mathrm{I}(2.13)$ \\
\hline 6 & $3(3.19)$ \\
\hline Recurrence rate & 33/94(35.11\%) \\
\hline
\end{tabular}


Table 3 Relationships Between the EORTC-GUCG Risk Scoring System and Clinicopathological Parameters of 94 Patients with NMIBC

\begin{tabular}{|c|c|c|c|c|c|}
\hline & All Patient $(n=94)$ & Low Risk (n=4I) & Intermediate Risk $(n=\mid 8)$ & High Risk $(n=35)$ & p-value \\
\hline \multicolumn{6}{|l|}{ Age(years) } \\
\hline Mean \pm SD & $63.22 \pm 12.28$ & $57.63 \pm 11.69$ & $67.44 \pm 15.60$ & $67.60 \pm 11.54$ & $<0.001$ \\
\hline Median (range) & $65(25-84)$ & $58(25-78)$ & $72(26-84)$ & $69(35-84)$ & \\
\hline \multicolumn{6}{|l|}{ Gender, n(\%) } \\
\hline Male & 75(79.79) & $35(85.37)$ & I4(77.78) & $26(74.29)$ & 0.474 \\
\hline Female & $19(20.21)$ & $6(14.63)$ & $4(22.22)$ & $9(25.7 I)$ & \\
\hline \multicolumn{6}{|l|}{ BMI, n(\%) } \\
\hline$<24$ & $57(60.64)$ & $26(63.4 I)$ & $10(55.56)$ & $21(60.00)$ & 0.847 \\
\hline$\geq 24$ & $37(39.36)$ & $15(36.59)$ & $8(44.44)$ & $14(40.00)$ & \\
\hline \multicolumn{6}{|l|}{ History of smoking, n(\%) } \\
\hline Yes & $52(55.32)$ & $19(46.34)$ & $\mathrm{II}(6 \mathrm{I} . \mathrm{II})$ & $22(62.86)$ & 0.303 \\
\hline No & $42(44.68)$ & $22(53.66)$ & $7(38.89)$ & $13(37.14)$ & \\
\hline \multicolumn{6}{|l|}{ Pathological T stage, n(\%) } \\
\hline pTa & $67(71.28)$ & $4 I(100.00)$ & $18(100.00)$ & $8(22.86)$ & $<0.001$ \\
\hline $\mathrm{PTI}$ & $27(28.72)$ & $0(0.00)$ & $0(0.00)$ & $27(77.14)$ & \\
\hline \multicolumn{6}{|l|}{ Tumor grade, n(\%) } \\
\hline LG & $58(61.70)$ & $4 I(100.0)$ & $16(88.89)$ & $\mathrm{I}(2.86)$ & $<0.001$ \\
\hline HG & $36(38.30)$ & $0(0.00)$ & $2(I I . I I)$ & $34(97.14)$ & \\
\hline \multicolumn{6}{|l|}{ Tumor size, n(\%) } \\
\hline$<3 \mathrm{~cm}$ & $57(60.64)$ & $4 I(100.00)$ & $2(11.11)$ & $14(40.00)$ & $<0.001$ \\
\hline$\geq 3 \mathrm{~cm}$ & $37(39.36)$ & $0(0.00)$ & $16(88.89)$ & $2 I(60.00)$ & \\
\hline
\end{tabular}

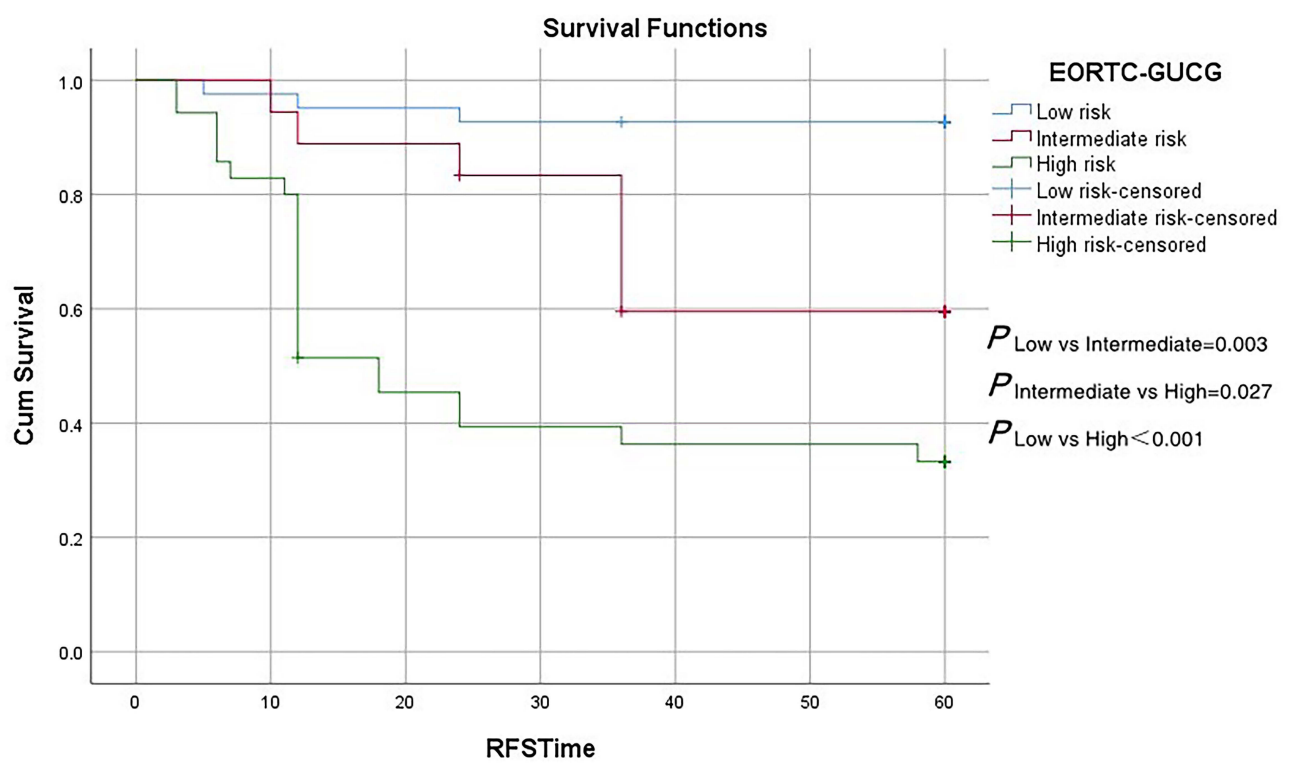

Figure I Survival curve of RFS in patients at risk for different EORTC-GUCG risk scores. 


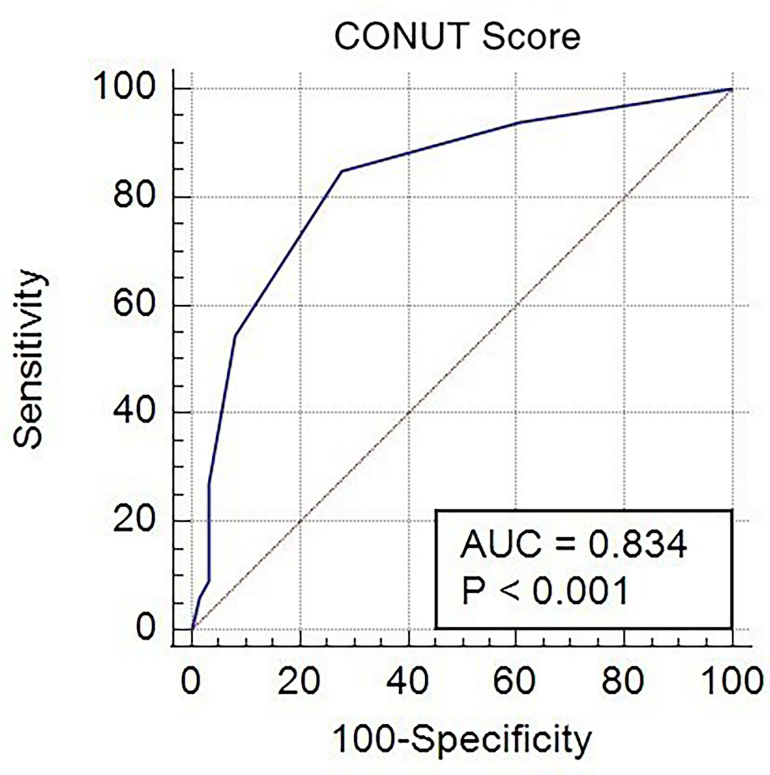

Figure 2 ROC curve of predictive value of the CONUT score for RFS in patients with NMBIC.

\section{Comparison of Recurrence Risk Among} Patients with Different EORTC-GUCG

\section{Risk Scores}

During the five-year follow-up period, 33 of 94 patients (38.0\%) experienced intravesical recurrence, including 3 cases $(7.32 \%)$ in the low-risk group, 7 cases $(38.89 \%)$ in the intermediate-risk group, and 23 cases $(65.71 \%)$ in the high-risk group. In addition, the Kaplan-Meier analysis demonstrated that the EORTC-GUCG risk scoring system had a significant correlation with RFS, and the high-risk group patients had shorter RFS than the other two groups $(\mathrm{p}<0.05)$ (Figure 1).

\section{Optimal Cut-off and Prognostic Values in} Predicting RFS of Preoperative CONUT

\section{Score}

The median preoperative serum albumin level was 41.99 (33.36-50.20) $(\mathrm{g} / \mathrm{dL})$, the median total lymphocyte count was $1.60(0.43-3.04) \times 109(/ \mathrm{mm} 3)$, and the median total cholesterol was $170.46(111.72-217.87)(\mathrm{mg} / \mathrm{dL})$. The mean CONUT score was 1.49 in this study. An ROC curve analysis showed that the optimal cut-off value of the CONUT score was 1 , which provided $84.85 \%$ sensitivity, $72.13 \%$ specificity, and the AUROC curves of CONUT score for RFS evaluation was 0.834 (Figure 2). Based on the cut-off value, 45 (47.87\%) patients were
Table 4 Clinic-Pathological Characteristics Comparison of 94 Patients with NMIBC Stratified by CONUT

\begin{tabular}{|c|c|c|c|}
\hline & CONU & Score & p-value \\
\hline & Low $(n=49)$ & High $(n=45)$ & \\
\hline Age(years) & & & \\
\hline Mean $\pm S D$ & $63.37 \pm|0.7|$ & $66.33 \pm 15.12$ & 0.029 \\
\hline Median (range) & $65(25-84)$ & $69(35-84)$ & \\
\hline Gender, n(\%) & & & \\
\hline Male & $39(79.59)$ & $36(80.00)$ & 0.961 \\
\hline Female & $10(20.4 I)$ & $9(20.00)$ & \\
\hline BMI, n(\%) & & & \\
\hline$<24$ & $26(53.06)$ & $3 I(68.89)$ & 0.117 \\
\hline$\geq 24$ & $23(46.84)$ & $14(31.1 \mid)$ & \\
\hline $\begin{array}{l}\text { History of smoking, } n \\
\text { (\%) }\end{array}$ & & & \\
\hline No & $23(46.94)$ & $19(42.22)$ & 0.646 \\
\hline Yes & $26(53.06)$ & $26(57.78)$ & \\
\hline $\begin{array}{l}\text { Pathological T stage, } n \\
\text { (\%) }\end{array}$ & & & \\
\hline pTa & $45(91.84)$ & $22(48.89)$ & $<0.001$ \\
\hline PTI & $4(8.16)$ & $23(51.11)$ & \\
\hline Tumor grade, $n(\%)$ & & & \\
\hline LG & $43(87.76)$ & $15(33.33)$ & $<0.001$ \\
\hline HG & $6(12.24)$ & $30(66.67)$ & \\
\hline Tumor size, $n(\%)$ & & & \\
\hline$<3 \mathrm{~cm}$ & $40(81.63)$ & 17(37.78) & $<0.001$ \\
\hline$\geq 3 \mathrm{~cm}$ & $9(18.37)$ & $28(62.22)$ & \\
\hline Serum albumin $(g / d L)$ & $43.98 \pm 3.46$ & $39.83 \pm 3.92$ & $<0.001$ \\
\hline $\begin{array}{l}\text { Total Lymphocyte count } \\
\text { (/mm3) }\end{array}$ & $1.90 \pm 0.41$ & $1.27 \pm 0.39$ & $<0.001$ \\
\hline $\begin{array}{l}\text { Total cholesterol } \\
(\mathrm{mg} / \mathrm{dL})\end{array}$ & $183.60 \pm 19.58$ & $156.15 \pm 20.66$ & $<0.001$ \\
\hline
\end{tabular}

classified as a high-CONUT group (>1) and 49 patients $(52.13 \%)$ were classified as a low-CONUT group (0-1).

The relationships between clinicopathological features and the CONUT score are shown in Table 4. Age, pathological T-stage, tumor size and grade were found to be significantly related with the preoperative CONUT score. Overall, the 1-year, 3-year, and 5-year RFS rates in patients with NMIBC were $76.60 \%$ (72/94), 64.44\% (58/ 90 ), and $63.33 \%$ (57/90), respectively. The mean time of recurrence (SD) was 17.30 (12.58) months. In addition, RFS were identified at 1-, 3-, and 5-year based on preoperative CONUT stratification in the study: 1-year RFS 


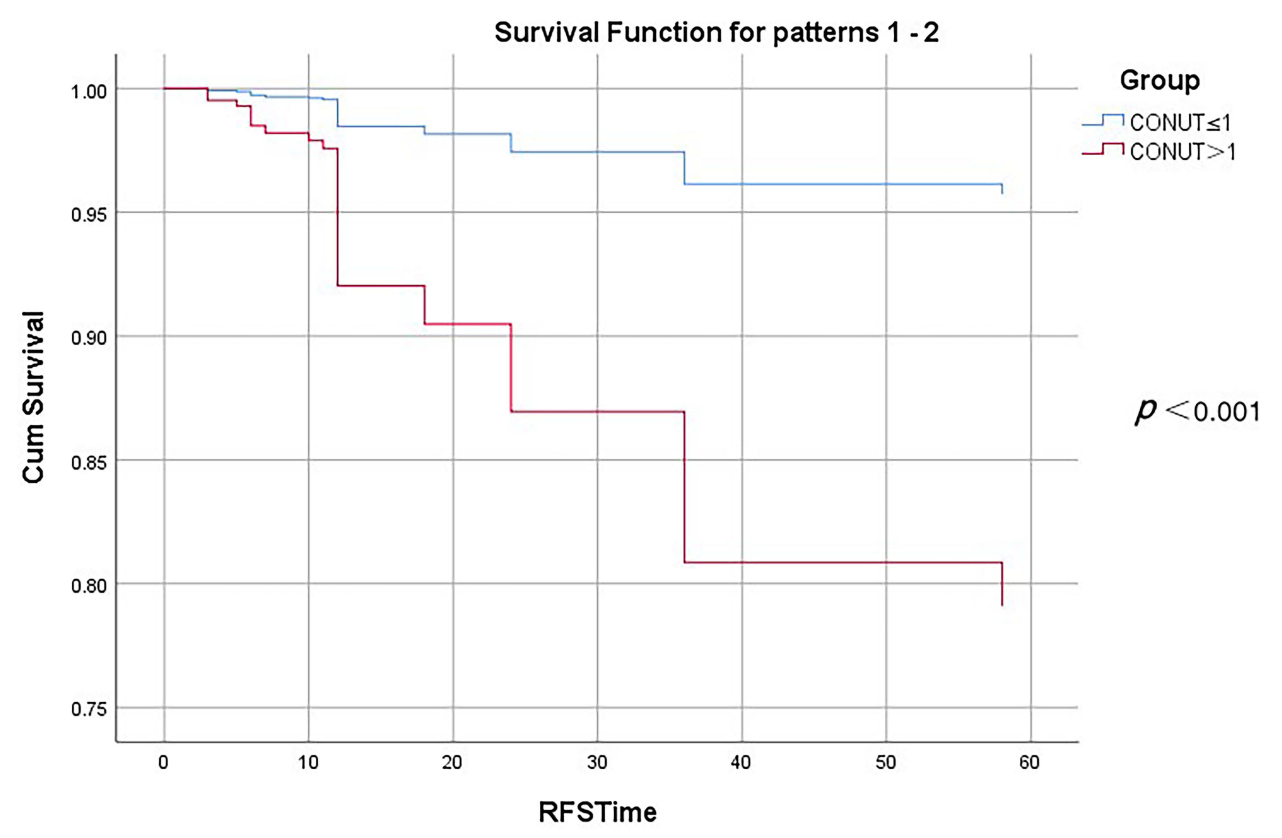

Figure 3 Survival curve of RFS in patients at different CONUT patients.

rate was $95.92 \%(47 / 49)$ in low-CONUT group and $55.56 \%(25 / 45)$ in high-CONUT group $(\mathrm{p}<0.001)$; 3 -year RFS rate was $89.80 \%(44 / 49)$ in low-CONUT group and $34.15 \%(14 / 41)$ in high-CONUT group $(\mathrm{p}<0.001)$; and 5-year RFS rate was $89.80 \%(44 / 49)$ in low-CONUT group and $31.71 \%(13 / 41)$ in high-CONUT group $(\mathrm{p}<0.001)$. The Kaplan-Meier survival analysis revealed a significant association between the preoperative CONUT score and RFS in patients $(\mathrm{p}<0.001$, Figure 3$)$. Therefore, high preoperative CONUT score could predict poorer RFS. In addition, we evaluated whether the CONUT score in different EORTC-GUCG groups was associated with RFS, and the results showed that there was no significant difference for RFS between the low-risk group $(p=0.242$, Figure $4 \mathrm{~A}$ ) and intermediate-risk ( $\mathrm{p}=0.165$, Figure $4 \mathrm{~B})$, while the difference in RFS was statistically significant in the high-risk groups ( $\mathrm{p}=0.043$, Figure $4 \mathrm{C}$ ).

In univariate analysis, RFS had no difference in gender and BMI. Age $(<65$ or $\geq 65$ ), history of smoking (yes or no), pathological T stage (pTa or pT1), tumor grade (LG or $\mathrm{HG}$ ), tumor size $(<3$ or $\geq 3 \mathrm{~cm})$, and preoperative CONUT score $(\leq 1$ or $>1)$ were found to be significantly associated with RFS (Table 5). In order to determine the independent prognostic value of the CONUT score, significant variables in univariate analysis were used as covariates for multivariate Cox proportional hazards regression analysis. Consequently, the results showed that tumor grade (LG or HG; HR $=3.056$, 95\% CI 1.030-9.069, p=0.044), tumor size $(<3$ or $\geq 3 \mathrm{~cm} ; \mathrm{HR}=2.733,95 \%$ CI $1.148-6.508$, $\mathrm{p}=0.023)$ and CONUT score $(\leq 1$ or $>1$; HR $=3.855,95 \%$ CI 1.242-11.970, $\mathrm{p}=0.020)$ were independent predictors of RFS in patients (Table 5).

\section{Development of a Nomogram for Predicting RFS in Patients with NMIBC}

In the multivariable Cox model, RFS of patients with NMIBC was significantly affected by tumor grade, pathological $\mathrm{T}$ stage and the preoperative CONUT score, and were consequently included in the nomogram to predict 1-, 3- and 5-year RFS rates in patients with NMIBC following primary surgery (Figure 5). In addition, according to the recommendations of relevant guidelines ${ }^{21,22}$ and previous models, ${ }^{23-27}$ the patient's age, history of smoking, pathological $\mathrm{T}$ stage were also incorporated into the nomogram to improve its forecasting ability. The c-index of the prediction model is 0.851 , which indicated that the prediction accuracy was relatively high. Meanwhile, it was also important that the calibration plots revealed a limited deviation from the ideal prediction by internal validation (Figure 6).

\section{Discussion}

NMBIC is the most prevalent pathological tumor-type in patients with initially diagnosed bladder cancer, but many patients may relapse, even after radical surgery. ${ }^{28}$ There is 

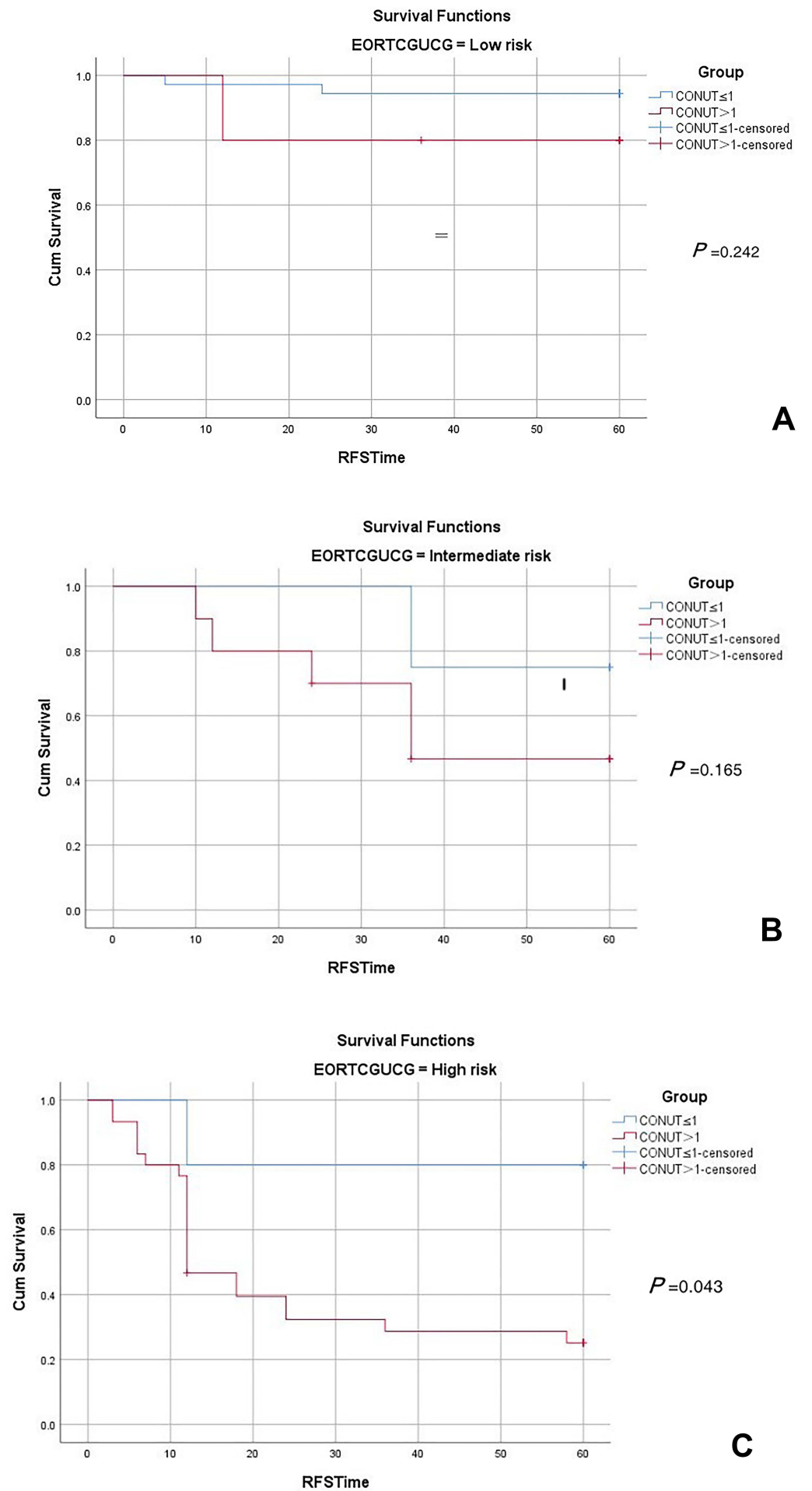

Figure 4 Survival curves of RFS in different CONUT patients with different EORTC-GUCG scores: (A) low-risk group; (B) intermediate-risk group; and (C) high-risk group.

evidence that cystectomy for patients who progress from NMIBC to muscle invasive bladder cancer (MIBC) have a poor prognosis in comparison to patients initially diagnosed with MIBC. ${ }^{29}$ Result predictions based solely on physician experience may be subjectively influenced. Therefore, it is of great significance to construct a simple and efficient prediction method for early detection and diagnosis of recurrent bladder cancer while improving 
the principles of follow-up and treatment plan. Only by accurately predicting the progress risk of patients with NMIBC can we formulate the best individualized treatment and monitoring plan for newly diagnosed and relapsed patients, but the tools for evaluating the progress risk are still insufficient at present.

In the past few years, two European organizations have developed two predictive models for assessing the risk of recurrence and progression of patients with NMIBC: the EORTC and CUETO score models. The EORTC-GUCG risk system is one of the commonly used models to evaluate the prognosis of patients, with a high predictive efficiency. ${ }^{30}$ In this study, pathological factors as tumor size, grade and T stage were the main risk factors affecting EORTC-GUCG score in patients with bladder cancer, which was consistent with the conclusions of Busato, ${ }^{31}$ Cerbone $^{32}$ and other studies. Following patient stratification through the EORTC-GUCG risk scoring system, the results revealed statistically significant differences in RFS outcomes $(\mathrm{p}<0.05)$ among the different groups, suggesting the accuracy of our patient datasets. Further determination of the optimal cut-off value of CONUT score led to our results revealing that the high-CONUT group had a lower rate of RFS than the low CONUT group, while the CONUT score was an independent prognostic factor associated with RFS, as validated by the multivariate Cox proportional hazards regression analysis.

The nutrition and inflammatory status of cancer patients can potentially predict post-surgical prognosis, and also affects the progression of malignant tumor, response to anti-cancer treatment, length of hospital stay and cost. Perioperative nutritional support for malnourished cancer patients can improve systemic nutritional status, enhance the tolerance levels during therapy and have a positive impact on postoperative survival. ${ }^{33,34}$ The CONUT score was initially reported as an effective tool for early detection and ongoing control of malnutrition in hospitals. ${ }^{12}$ With the deepening of its research, people found that the CONUT score has significant prognostic value in various malignant tumors, such as esophageal, ${ }^{13}$ renal, ${ }^{14}$ gastric, ${ }^{15}$ prostate, ${ }^{16}$ and colorectal cancer. $^{17}$ However, no study has systematically identified the prognostic effect of preoperative CONUT score on posttreatment RFS in patients with NMBIC cancer.

Among the three components of the CONUT score, the concentration of serum albumin is the most important parameter, scoring two-fold higher in comparison to the other two parameters. Serum albumin concentration reflects nutritional status and is a reliable indicator of inflammation, chronic disease and humoral conditions. ${ }^{35}$ It is associated with tumor necrosis since C-Reactive Protein (CRP) and pro-inflammatory cytokines, such as TNF- $\alpha$ or IL-6, can reduce albumin synthesis by modulating hepatocytes. ${ }^{36}$ Consequently, it has been reported that hypoalbuminemia is closely associated with cancer survival in the literature, and perioperative nutritional support is considered to help improve surgical outcomes in patients. ${ }^{10}$ Total lymphocyte count is an important indicator of the immunological and nutritional status of body, and it is the main cellular component of the immune system, which can produce immune responses to tumor cells. $^{37}$ The decrease in lymphocytes will lead to a reduction in such anti-tumor immune responses. Mella et $\mathrm{al}^{38}$ have found that a decrease in $\mathrm{T}$ cell count is associated with a poor prognosis in tumor patients since the host had a compromised immunity to cancer cells. Therefore, a low peripheral blood lymphocyte count reflects an insufficient host immune response and can be used as an indicator of poor prognosis for various tumors. $^{39-41}$

CONUT score is different from other scores in introducing serum total cholesterol level. Cholesterol is one of the most important components of cell membrane. In addition to tumorigenesis, cholesterol is also related to many potential biochemical pathways related to immune response, ${ }^{42,43}$ such as it could increase the antigen-presenting function of monocytes. ${ }^{44}$ Compared with hypercholesterolemia, hypocholesterolemia is more related to the decrease of peripheral circulating lymphocytes, total T cells and CD8+ cells. Cholesterol. Therefore, low serum total cholesterol levels can affect intracellular signal transduction and impair the immune system, resulting in poor prognosis. Meanwhile, the transcriptomic expression level of low density lipoprotein receptor within tumor tissue is upregulated. ${ }^{45}$ It indicates that the tumor tissue exacerbates intake of low-density lipoprotein cholesterol, for the purpose of accelerating tumor growth and consequently consuming serum cholesterol levels. ${ }^{46}$ This justifies why cholesterol levels typically rise after surgery. In summary, low serum total cholesterol level is not only considered to be a causative factor for cancer but also is regarded as a repercussion of cancer. In addition, total cholesterol concentration is considered to be an indicator of reserve calories in patients. $^{47}$ Reduction in serum cholesterol level is also reflected by a decrease in caloric intake. Consequently, it has been widely reported in the literature that cholesterol levels are related to tumor progression and cancer survival. ${ }^{48}$ Finally, 
Table 5 Univariate and Multivariate Cox Proportional Hazards Regression Analyses of RFS in 94 Patients with NMIBC

\begin{tabular}{|c|c|c|c|c|}
\hline \multirow[t]{2}{*}{ Variable } & \multicolumn{2}{|c|}{ Univariate } & \multicolumn{2}{|c|}{ Multivariate } \\
\hline & HR(95\% Cl) & p-value & HR(95\% Cl) & p-value \\
\hline $\begin{array}{l}\text { Age(years) } \\
\quad<65 \\
\geq 65\end{array}$ & $\begin{array}{c}\text { I (reference) } \\
3.25 \mathrm{I}(\mathrm{I} .509-7.004)\end{array}$ & 0.003 & I.43I (0.599-3.4I9) & 0.420 \\
\hline $\begin{array}{l}\text { Gender, } \mathrm{n}(\%) \\
\text { Male } \\
\text { Female }\end{array}$ & $\begin{array}{c}\text { I (reference) } \\
\text { I.307(0.590-2.899) }\end{array}$ & 0.509 & & \\
\hline $\begin{array}{l}\text { BMI, n(\%) } \\
\quad<24 \\
\geq 24\end{array}$ & $\begin{array}{c}\mathrm{I}(\text { reference }) \\
0.618(0.294-\mathrm{I} .298)\end{array}$ & 0.203 & & \\
\hline $\begin{array}{l}\text { History of smoking, n(\%) } \\
\text { No } \\
\text { Yes }\end{array}$ & $\begin{array}{c}\text { I (reference) } \\
2.547(1.182-5.487)\end{array}$ & 0.017 & $2.143(0.949-4.838)$ & 0.067 \\
\hline $\begin{array}{l}\text { Pathological T stage, } n(\%) \\
\text { PTa } \\
\text { PTI }\end{array}$ & $\begin{array}{c}\text { I (reference) } \\
3.540(I .78 I-7.033)\end{array}$ & $<0.001$ & $1.575(0.558-4.445)$ & 0.391 \\
\hline $\begin{array}{l}\text { Tumor grade, } n(\%) \\
\text { LG } \\
\text { HG }\end{array}$ & $\begin{array}{c}\text { I (reference) } \\
3.092(2.05 \mid-4.662)\end{array}$ & $<0.001$ & $3.056(1.030-9.069)$ & 0.044 \\
\hline $\begin{array}{l}\text { Tumor size, } \mathrm{n}(\%) \\
\quad<3 \mathrm{~cm} \\
\geq 3 \mathrm{~cm}\end{array}$ & $\begin{array}{c}\text { I (reference) } \\
5.5 \mathrm{II}(2.547-1 \mathrm{I} .924)\end{array}$ & $<0.001$ & $2.733(1.148-6.508)$ & 0.023 \\
\hline $\begin{array}{l}\text { CONUT } \\
\leq 1 \\
>1\end{array}$ & $\begin{array}{c}\text { I (reference) } \\
8.864(3.400-23.105)\end{array}$ & $<0.001$ & $3.855(1.242-11.970)$ & 0.020 \\
\hline
\end{tabular}

through the combination of the above three parameters, the accuracy of each parameter can be integrated to better assess the general situation of the patients.

The CONUT score is not the only index for assessing nutrition, prognostic nutritional index (PNI), neutrophil-lymphocyte ratio (NLR) and Glasgow prognostic score (GPS) have been also employed to evaluate the general situation of patients and have showed to be associated with cancer survival. ${ }^{49-51}$ However, the majority of studies have reported that the CONUT score was the most accurate indicator for predicting prognosis. $^{52-54}$ In GPS calculations, although levels of CRP are closely related to systemic inflammatory responses, false-positive results could occur in the earlyphase of infection or immunodeficient hosts. Similar to CONUT scores, serum albumin and total lymphocyte counts are also used in the calculation of PNI, which may explain the similar AUCs results of CONUT and
PNI. But unlike PNI, COUNT scores include total cholesterol as a variable, as mentioned above, hypocholesterolemia has a unique role in the response to malnutrition and autoimmune diseases in cancer patients. Through comparison of the CONUT and PNI scoring systems, Takagi et $\mathrm{al}^{55}$ show that the CONUT scoring system is more superior to PNI in predicting the survival of patients with various malignancies.

Unlike the inclusion of relapsed cases $(44.3 \%)$ in the EORTC cohort, only patients with NMIBC who were initially diagnosed and had no previous relapse were included in our cohort study. This enrollment was also noted in previous studies. ${ }^{8,25,56}$ Compared to these models for predicting NMBIC recurrence, our nomogram has a higher prediction accuracy on internal validation $(\mathrm{C}$-index $=0.851)$, although the prediction accuracy of this nomogram has not been externally validated. In this study, we explored the relationship between the CONUT score 


Points
Age
History_of_smoking
Tumor_stage
Tumor_grade
Tumor_size
Conut
Total Points
Linear Predictor
1-year RFS
3-year RFS
5-year RFS

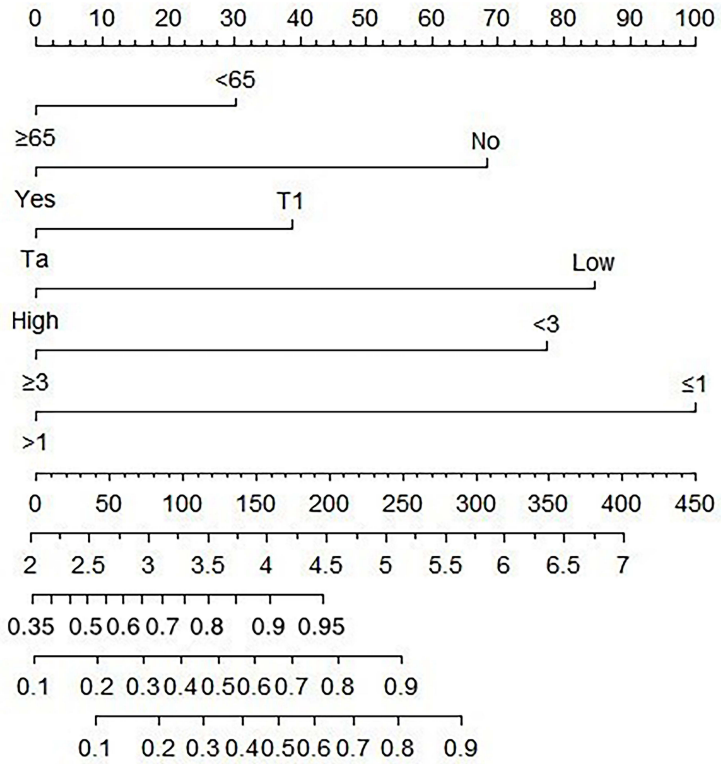

Figure 5 Nomograms to predict RFS rates at I-, 3- and 5-year in patients with NMIBC.

and prognosis of patients with NMBIC who had underwent radical resection, demonstrating that preoperative CONUT score was an independent prognostic factor for postoperative RFS in patients. This is the first report that CONUT acted as an independent predictor, combined with the statistically significant parameters of multivariate analysis, to evaluate the prognosis of patients with NMIBC in a single prediction model. Furthermore, we also included well-established predictors of tumor relapse to increase the discriminant properties of our nomograms, which has identified in the previous guidelines ${ }^{21,22}$ and predictive models. ${ }^{23-27}$ Such properties included age, history of smoking and $\mathrm{T}$ stage, though they did not show obvious significance in the multivariate analysis (this may be due to the patient enrollment paucity in the study cohort).

Although our findings were significant, our limitations were equally apparent. First of all, our research was a retrospective analysis of a single-center design, which may lead to inherent bias. There are various forms of postoperative adjuvant therapies for patients, and the treatment process was not unified. Due to the limitations of retrospective analysis, it is difficult to trace the exact information of the postoperative intravesical treatment regimen (eg, type and dose of chemotherapy drugs; $\mathrm{MMC/}$ epirubicin/gemcitabine /etc). Secondly, other potential prognostic factors that were not included in our study variables, such as tumor focality, preoperative positive urine cytology, and the presence of CIS, which have been regarded as important prognostic factors in recent studies ${ }^{26,28,57}$ and can be added as parameters to the nomogram to improve the effectiveness in clinical practice. Thirdly, our sample size could not be considered as sufficient. The limited number of patients inhibited the selection of parameters in our nomogram and the results of internal validation. Fourthly, the nomograms generated in this study were solely validated internally only and were not validated externally in large multicenter cohort studies to determine its validity in clinical prediction. Lastly, potential factors such as medication (eg, statins) and nutritional support that might have influenced the inflammatory and nutritional parameters of the CONUT score were not considered in this study.

Our novel nomogram based on CONUT score constructed by us in a single-institution database may not be completely accurate, but it showed a reasonable level of identification through internal validation. Therefore, our nomogram can be used as another predictive tool to predict tumor recurrence in patients with NMIBC following TURBT or partial cystectomy. In order to generalize the applicability of the developed nomogram in actual clinical practice, further validation needs to be performed using other larger, multicenter external patient cohorts.

\section{Conclusions}

This study explored the value of preoperative CONUT score for the prediction of RFS in patients with NMIBC, and confirmed that the CONUT score is an independent prognostic factor for RFS. Patients with a high CONUT score had an elevated risk of recurrence in comparison to patients with a low CONUT score. Furthermore, we 

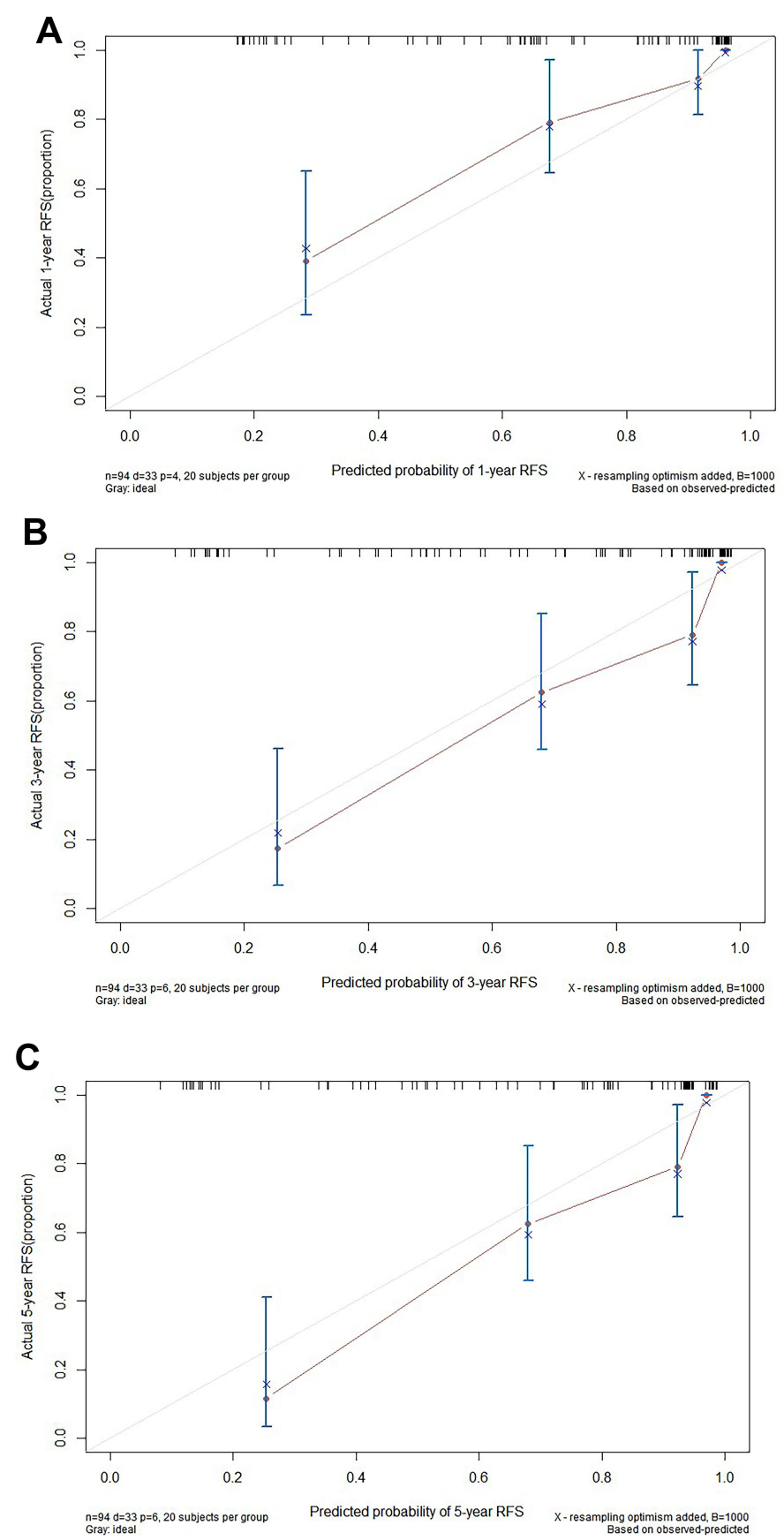

Figure 6 Internal validation of calibration curve for RFS evaluation in patients with NMIBC at I- (A) 3- (B) and 5-year (C).

provided a nomogram for evaluate the probability of tumor recurrence in patients with NMIBC who have underwent TURBT or partial cystectomy, with internal validation showing reasonable calibration. The development of a new nomogram, based on preexisting prognostic parameters in combination with CONUT, could increase the accuracy of recurrence prediction and improve individualized therapy for patients with NMIBC.

\section{Abbreviations}

BMI, Body Mass Index; HG, high grade; LG, low grade; CONUT, Controlling Nutritional Status; NMIBC, non-muscle-invasive bladder cancer; EORTCGUCG, European Organization for the Research and Treatment of Cancer; SD, standard deviation; RFS, recurrence-free survival; HR, hazard ratio; CI, confidence interval. 


\section{Ethics Approval and Consent to Participate}

All procedures were performed in accordance with the Declaration of Helsinki of the World Medical Association. The scheme was authorized by Ethics Committee of Zhejiang Xiaoshan Hospital.

\section{Author Contributions}

Liwei Zhao: conception and design of the work, literature review, drafting manuscript, analysis, and/or interpretation of the data; Ji Sun: design of the work and literature review, data collection and/or processing; Kai Wang: conception and design of the work, data collection and/or processing; Shengcheng Tai: data collection and/or processing; Runmiao Hua: data collection and/or processing; Yufu Yu: data collection and/or processing; Yi Fan: supervision and critical review; Jiaguo Huang: supervision and critical review. All authors contributed to data analysis, drafting or revising the article, have agreed on the journal to which the article will be submitted, gave final approval of the version to be published, and agree to be accountable for all aspects of the work.

\section{Funding}

There is no funding to report.

\section{Disclosure}

Authors declare no conflicts of interest for this article.

\section{References}

1. Antoni S, Ferlay J, Soerjomataram I, Znaor A, Jemal A, Bray F. Bladder cancer incidence and mortality: a global overview and recent trends. Eur Urol. 2017;71(1):96-108. Epub 2016 Jun 28. PMID: 27370177. doi:10.1016/j.eururo.2016.06.010

2. Jemal A, Bray F, Center MM, Ferlay J, Ward E, Forman D. Global cancer statistics. CA Cancer J Clin. 2011;61(2):69-90. Epub 2011 Feb 4. Erratum in: CA Cancer J Clin. 2011 Mar-Apr;61(2):134. PMID: 21296855. doi:10.3322/caac.20107

3. Ferlay J, Shin HR, Bray F, Forman D, Mathers C, Parkin DM. Estimates of worldwide burden of cancer in 2008: GLOBOCAN 2008. Int J Cancer 2010;127(12):2893-2917. PMID: 21351269. doi:10.1002/ijc.25516

4. Burger M, Catto JW, Dalbagni G, et al. Epidemiology and risk factors of urothelial bladder cancer. Eur Urol. 2013;63(2):234-241. Epub 2012 Jul 25. PMID: 22877502. doi:10.1016/j.eururo.2012.07.033

5. Kang M, Jeong CW, Kwak C, Kim HH, Ku JH. Preoperative neutrophil-lymphocyte ratio can significantly predict mortality outcomes in patients with non-muscle invasive bladder cancer undergoing transurethral resection of bladder tumor. Oncotarget. 2017;8 (8):12891-12901. PMID: 28039452; PMCID: PMC5355064. doi:10.18632/oncotarget.14179

6. Wang B, Li X, Ming S, et al. Combined extraperitoneal and transperitoneal laparoscopic extended partial cystectomy for the treatment of urachal carcinoma. J Endourol. 2016;30(3):280-285. Epub 2015 Dec 18. PMID: 26531333. doi:10.1089/end.2015.0423
7. Albayrak S, Zengin K, Tanik S, et al. Can the neutrophil-tolymphocyte ratio be used to predict recurrence and progression of non-muscle-invasive bladder cancer? Kaohsiung J Med Sci. 2016;32 (6):327-333. Epub 2016 Jun 2. PMID: 27377847. doi:10.1016/j. kjms.2016.05.001

8. Hong SJ, Cho KS, Han M, et al; Korean Urological Oncology Society. Nomograms for prediction of disease recurrence in patients with primary $\mathrm{Ta}, \mathrm{T} 1$ transitional cell carcinoma of the bladder. J Korean Med Sci. 2008;23(3):428-433. PMID: 18583878; PMCID: PMC2526537. doi:10.3346/jkms.2008.23.3.428

9. Black PC. Fine-tuning risk stratification for non-muscle-invasive bladder cancer. Eur Urol. 2016;69(1):70-71. PMID: 26256521. doi:10.1016/j.eururo.2015.07.034

10. Gupta D, Lis CG. Pretreatment serum albumin as a predictor of cancer survival: a systematic review of the epidemiological literature. Nutr J. 2010;9(1):69. PMID: 21176210; PMCID: PMC3019132. doi:10.1186/1475-2891-9-69

11. Hanahan D, Weinberg RA. Hallmarks of cancer: the next generation. Cell. 2011;144(5):646-674. PMID: 21376230. doi:10.1016/j. cell.2011.02.013

12. Ignacio de Ulíbarri J, González-Madroño A, de Villar NG, et al. CONUT: a tool for controlling nutritional status. First validation in a hospital population. Nutr Hosp. 2005;20(1):38-45. PMID: 15762418 .

13. Yoshida N, Baba Y, Shigaki H, et al. Preoperative nutritional assessment by Controlling Nutritional Status (CONUT) is useful to estimate postoperative morbidity after esophagectomy for esophageal cancer. World J Surg. 2016;40(8):1910-1917. PMID: 27220507. doi:10.1007/s00268-016-3549-3

14. Song H, Xu B, Luo C, et al. The prognostic value of preoperative controlling nutritional status score in non-metastatic renal cell carcinoma treated with surgery: a retrospective single-institution study. Cancer Manag Res. 2019;11:7567-7575. PMID: 31496811; PMCID: PMC6693320. doi:10.2147/CMAR.S209418

15. Hirahara N, Tajima Y, Fujii Y, et al. Controlling Nutritional Status (CONUT) as a prognostic immune nutritional biomarker for gastric cancer after curative gastrectomy: a propensity score-matched analysis. Surg Endosc. 2019;33(12):4143-4152. doi:10.1007/s00464-019-06723-Z

16. Zhang W, Wu Y, Zhang Z, et al. Controlling nutritional status score: a new prognostic indicator for patients with oligometastatic prostate cancer. Curr Probl Cancer. 2019;43(5):461-470. Epub 2019 Mar 15. PMID: 30910226. doi:10.1016/j.currproblcancer.2019.02.001

17. Takagi K, Buettner S, Ijzermans JNM. Prognostic significance of the controlling nutritional status (CONUT) score in patients with colorectal cancer: a systematic review and meta-analysis. Int $J$ Surg. 2020;78:91-96. Epub 2020 Apr 23. PMID: 32335238. doi:10.1016/j. ijsu.2020.04.046

18. Montironi R, Lopez-Beltran A. The 2004 WHO classification of bladder tumors: a summary and commentary. Int J Surg Pathol. 2005;13 (2):143-153. PMID: 15864376. doi:10.1177/106689690501300203

19. Paner GP, Stadler WM, Hansel DE, Montironi R, Lin DW, Amin MB. Updates in the eighth edition of the tumor-node-metastasis staging classification for urologic cancers. Eur Urol. 2018;73(4):560-569. Epub 2018 Jan 9. PMID: 29325693. doi:10.1016/j. eururo.2017.12.018

20. Sylvester RJ, van der Meijden AP, Oosterlinck W, et al. Predicting recurrence and progression in individual patients with stage $\mathrm{Ta} \mathrm{T} 1$ bladder cancer using EORTC risk tables: a combined analysis of 2596 patients from seven EORTC trials. Eur Urol. 2006;49(3):4665; discussion 475-7. Epub 2006 Jan 17. PMID: 16442208. doi:10.1016/j.eururo.2005.12.031

21. Cumberbatch MGK, Jubber I, Black PC, et al. Epidemiology of bladder cancer: a systematic review and contemporary update of risk factors in 2018. Eur Urol. 2018;74(6):784-795. Epub 2018 Sep 26. PMID: 30268659. doi:10.1016/j.eururo.2018.09.001 
22. Kluth LA, Black PC, Bochner BH, et al. Prognostic and prediction tools in bladder cancer: a comprehensive review of the literature. Eur Urol. 2015;68(2):238-253. Epub 2015 Feb 21. PMID: 25709027. doi: $10.1016 / j$.eururo.2015.01.032

23. Kim HS, Ku JH, Kim SJ, et al. Prognostic factors for recurrence and progression in Korean non-muscle-invasive bladder cancer patients: a Retrospective, Multi-Institutional Study. Yonsei Med J. 2016;57 (4):855-864. PMID: 27189277; PMCID: PMC4951460. doi:10.3349/ymj.2016.57.4.855

24. Sfakianos JP, Kim PH, Hakimi AA, Herr HW. The effect of restaging transurethral resection on recurrence and progression rates in patients with nonmuscle invasive bladder cancer treated with intravesical bacillus Calmette-Guérin. J Urol. 2014;191(2):341-345. Epub 2013 Aug 20. PMID: 23973518; PMCID: PMC4157345. doi:10.1016/j. juro.2013.08.022

25. Pan CC, Chang YH, Chen KK, Yu HJ, Sun CH, Ho DM. Constructing prognostic model incorporating the 2004 WHO/ISUP classification for patients with non-muscle-invasive urothelial tumours of the urinary bladder. $J$ Clin Pathol. 2010;63 (10):910-915. PMID: 20876324. doi:10.1136/jcp.2010.079764

26. Shindo T, Masumori N, Kitamura H, et al. Clinical significance of definite muscle layer in TUR specimen for evaluating progression rate in T1G3 bladder cancer: multicenter retrospective study by the Sapporo Medical University Urologic Oncology Consortium (SUOC). World J Urol. 2014;32(5):1281-1285. Epub 2013 Nov 5. PMID: 24190368. doi:10.1007/s00345-013-1205-1

27. Fernandez-Gomez J, Madero R, Solsona E, et al. Predicting nonmuscle invasive bladder cancer recurrence and progression in patients treated with bacillus Calmette-Guerin: the CUETO scoring model. $J$ Urol. 2009;182(5):2195-2203. Epub 2009 Sep 16. PMID: 19758621. doi:10.1016/j.juro.2009.07.016

28. Dearnaley D, Syndikus I, Mossop H, et al; CHHiP Investigators. Conventional versus hypofractionated high-dose intensity-modulated radiotherapy for prostate cancer: 5-year outcomes of the randomised, non-inferiority, Phase 3 CHHiP trial. Lancet Oncol. 2016;17 (8):1047-1060. Epub 2016 Jun 20. Erratum in: Lancet Oncol. 2016 Aug;17 (8): e321.PMID: 27339115; PMCID: PMC4961874. doi:10.1016/S1470-2045(16)30102-4

29. Liedberg F, Hagberg O, Holmäng S, et al. Local recurrence and progression of non-muscle-invasive bladder cancer in Sweden: a population-based follow-up study. Scand $J$ Urol. 2015;49 (4):290-295. Epub 2015 Jan 26. PMID: 25624049. doi:10.3109/ 21681805.2014.1000963

30. Kılınç MF, Bayar G, Dalkılıç A, Sönmez NC, Arısan S, Güney S. Applicability of the EORTC risk tables to predict outcomes in non-muscle-invasive bladder cancer in Turkish patients. Turk $J$ Urol. 2017;43(1):48-54. Epub 2017 Jan 6. PMID: 28270951; PMCID: PMC5330268. doi:10.5152/tud.2016.77603

31. Busato Júnior WF, Almeida GL, Ribas CA, Ribas Filho JM, De Cobelli O. EORTC risk model to predict progression in patients with non-muscle-invasive bladder cancer: is it safe to use in clinical practice? Clin Genitourin Cancer. 2016;14(2):176-182. Epub 2015 Sep 25. PMID: 26444918. doi:10.1016/j.clgc.2015.09.005

32. Cerbone L, Sternberg CN, Sengeløv L, et al. Results from a phase I Study of lapatinib with gemcitabine and cisplatin in advanced or metastatic bladder cancer: EORTC trial 30061. Oncology. 2016;90 (1):21-28. Epub 2015 Oct 16. PMID: 26468947. doi:10.1159/ 000440959

33. Ali Abdelhamid Y, Chapman MJ, Deane AM. Peri-operative nutrition. Anaesthesia. 2016;71(Suppl 1):9-18. PMID: 26620142. doi:10.1111/anae. 13310

34. Liu Y, Xue X. Systematic review of peri-operative nutritional support for patients undergoing hepatobiliary surgery. Hepatobiliary Surg Nutr. 2015;4(5):304-312. PMID: 26605277; PMCID: PMC4607833. doi:10.3978/j.issn.2304-3881.2014.12.09
35. Ballmer PE. Causes and mechanisms of hypoalbuminaemia. Clin Nutr. 2001;20(3):271-273. PMID: 11407876. doi:10.1054/ clnu.2001.0439

36. Peters SJ, Vanhaecke T, Papeleu P, Rogiers V, Haagsman HP, van Norren K. Co-culture of primary rat hepatocytes with rat liver epithelial cells enhances interleukin-6-induced acute-phase protein response. Cell Tissue Res. 2010;340(3):451-457. Epub 2010 Apr 22. PMID: 20411395; PMCID: PMC2882052. doi:10.1007/ s00441-010-0955-y

37. Cohen S, Danzaki K, MacIver NJ. Nutritional effects on T-cell immunometabolism. Eur J Immunol. 2017;47(2):225-235. Epub 2017 Jan 24. PMID: 28054344; PMCID: PMC5342627. doi:10.1002/eji.201646423

38. Mella M, Kauppila JH, Karihtala P, et al. Tumor infiltrating CD8+ T lymphocyte count is independent of tumor TLR9 status in treatment naïve triple negative breast cancer and renal cell carcinoma. Oncoimmunology. 2015;4(6):e1002726. PMID: 26155410; PMCID: PMC4485740. doi:10.1080/2162402X.2014.1002726

39. Karantanos T, Karanika S, Seth B, Gignac G. The absolute lymphocyte count can predict the overall survival of patients with non-small cell lung cancer on nivolumab: a clinical study. Clin Transl Oncol. 2019;21(2):206-212. Epub 2018 Jun 13. PMID: 29948974. doi:10.1007/s12094-018-1908-2

40. Vicente Conesa MA, Garcia-Martinez E, Gonzalez Billalabeitia E, et al. Predictive value of peripheral blood lymphocyte count in breast cancer patients treated with primary chemotherapy. Breast. 2012;21 (4):468-474. Epub 2011 Nov 26. PMID: 22119767. doi:10.1016/j. breast.2011.11.002

41. Hoskin PJ, Rojas AM, Peiris SN, Mullassery V, Chong IY. Pretreatment haemoglobin and peripheral blood lymphocyte count as independent predictors of outcome in carcinoma of cervix. Clin Oncol. 2014;26(4):179-184. Epub 2014 Jan 15. PMID: 24439272. doi:10.1016/j.clon.2013.11.023

42. Wang C, Li P, Xuan J, et al. Cholesterol enhances colorectal cancer progression via ROS elevation and MAPK signaling pathway activation. Cell Physiol Biochem. 2017;42(2):729-742. Epub 2017 Jun 15. PMID: 28618417. doi:10.1159/000477890

43. Strasak AM, Pfeiffer RM, Brant LJ, et al; VHM\&PP Study Group. Timedependent association of total serum cholesterol and cancer incidence in a cohort of 172,210 men and women: a prospective 19-year follow-up study. Ann Oncol. 2009;20(6):1113-1120. Epub 2009 Jan 22. PMID: 19164459; PMCID: PMC2685450. doi:10.1093/annonc/mdn736

44. Hughes DA, Townsend PJ, Haslam PL. Enhancement of the antigen-presenting function of monocytes by cholesterol: possible relevance to inflammatory mechanisms in extrinsic allergic alveolitis and atherosclerosis. Clin Exp Immunol. 1992;87(2):279-286. PMID: 1370928; PMCID: PMC1554263. doi:10.1111/j.1365-2249.1992. tb02988.x

45. Gabitova L, Gorin A, Astsaturov I. Molecular pathways: sterols and receptor signaling in cancer. Clin Cancer Res. 2014;20(1):28-34. Epub 2013 Oct 24. PMID: 24158702; PMCID: PMC3859141. doi:10.1158/1078-0432.CCR-13-0122

46. Cruz PM, Mo H, McConathy WJ, Sabnis N, Lacko AG. The role of cholesterol metabolism and cholesterol transport in carcinogenesis: a review of scientific findings, relevant to future cancer therapeutics. Front Pharmacol. 2013;4:119. PMID: 24093019; PMCID: PMC3782849. doi:10.3389/fphar.2013.00119

47. Gadgil MD, Anderson CA, Kandula NR, Kanaya AM. Dietary patterns are associated with metabolic risk factors in South Asians living in the United States. J Nutr. 2015;145(6):1211-1217. Epub 2015 Apr 22. PMID: 25904730; PMCID: PMC4442115. doi:10.3945/jn.114.207753

48. Zhou P, Li B, Liu B, Chen T, Xiao J. Prognostic role of serum total cholesterol and high-density lipoprotein cholesterol in cancer survivors: a systematic review and meta-analysis. Clin Chim Acta. 2018;477:94-104. Epub 2017 Dec 6. PMID: 29223765. doi:10.1016/j.cca.2017.11.039 
49. Sakai M, Sohda M, Saito H, et al. Comparative analysis of immunoinflammatory and nutritional measures in surgically resected esophageal cancer: a Single-center Retrospective Study. In Vivo (Brooklyn). 2020;34(2):881-887. PMID: 32111799; PMCID: PMC7157860. doi:10.21873/invivo. 11853

50. Qiu X, Song Y, Cui Y, Liu Y. Increased neutrophil-lymphocyte ratio independently predicts poor survival in non-metastatic triple-negative breast cancer patients. IUBMB Life. 2018;70(6):529-535. Epub 2018 Apr 29. PMID: 29707892. doi:10.1002/iub.1745

51. Zhu M, Feng M, He F, et al. Pretreatment neutrophil-lymphocyte and platelet-lymphocyte ratio predict clinical outcome and prognosis for cervical cancer. Clin Chim Acta. 2018;483:296-302. Epub 2018 May 22. PMID: 29758203. doi:10.1016/j.cca.2018.05.025

52. Toyokawa T, Kubo N, Tamura T, et al. The pretreatment Controlling Nutritional Status (CONUT) score is an independent prognostic factor in patients with resectable thoracic esophageal squamous cell carcinoma: results from a retrospective study. BMC Cancer. 2016;16(1):722. PMID: 27599460; PMCID: PMC5013653. doi:10.1186/s12885-016-2696-0

53. Hirahara N, Matsubara T, Hayashi H, Takai K, Nakada S, Tajima Y. Prognostic importance of controlling nutritional status in patients undergoing curative thoracoscopic esophagectomy for esophageal cancer. Am J Ther. 2018;25(5):e524-e532. PMID: 26866437; PMCID: PMC6133196. doi:10.1097/MJT.0000000000000414
54. Hikage M, Taniyama Y, Sakurai T, et al. The influence of the perioperative nutritional status on the survival outcomes for esophageal cancer patients with neoadjuvant chemotherapy. Ann Surg Oncol. 2019;26(13):4744-4753. Epub 2019 Aug 22. PMID: 31440925. doi:10.1245/s10434-019-07742-9

55. Takagi K, Buettner S, Ijzermans JNM, Wijnhoven BPL. Systematic Review on the Controlling Nutritional Status (CONUT) score in patients undergoing esophagectomy for esophageal cancer. Anticancer Res. 2020;40(10):5343-5349. PMID: 32988852. doi:10.21873/anticanres.14541

56. Ieda T, Muto S, Shimizu F, et al. Development and validation of a novel recurrence risk stratification for initial non-muscle invasive bladder cancer in Asia. EBioMedicine. 2016;12:98-104. Epub 2016 Sep 2. PMID: 27614395; PMCID: PMC5078579. doi:10.1016/j. ebiom.2016.08.051

57. Ali-El-Dein B, Sooriakumaran P, Trinh QD, Barakat TS, Nabeeh A. Construction of predictive models for recurrence and progression in $>1000$ patients with non-muscle-invasive bladder cancer (NMIBC) from a single centre. BJU Int. 2013;111(8):E331-E341. Epub 2013 Feb 27. PMID: 23445082. doi:10.1111/bju.12026

\section{Publish your work in this journal}

Cancer Management and Research is an international, peer-reviewed open access journal focusing on cancer research and the optimal use of preventative and integrated treatment interventions to achieve improved outcomes, enhanced survival and quality of life for the cancer patient.
The manuscript management system is completely online and includes a very quick and fair peer-review system, which is all easy to use. Visit http://www.dovepress.com/testimonials.php to read real quotes from published authors. 